\title{
Merging Monte Carlo and Dynamical EBSD Simulations
}

\author{
P.G. Callahan ${ }^{1}$ and M. De Graef ${ }^{1}$ \\ 1. Department of Materials Science and Engineering, Carnegie Mellon Univ., Pittsburgh PA 15213
}

Electron back-scatter diffraction (EBSD) patterns provide information about the orientation of the crystal lattice at the point of incidence of the electron beam on a highly inclined sample. The positions and orientations of Kikuchi lines can be converted into an Euler angle triplet or a unit quaternion, and repeating this procedure as the beam is scanned across the sample leads to orientation maps. While this aspect of EBSD work is well understood and commercially available in several implementations, the detailed intensity distribution of the patterns are a bit more difficult to understand and model. Each incident electron undergoes a stochastic series of elastic and inelastic scattering processes, changing direction and losing energy along the way; a portion of these electrons exit the sample surface in the general direction of the scintillator. Monte Carlo (MC) trajectory simulations were used to model the spatial and energy distribution of BSEs for a common scintillator and sample geometry. The energy distribution of electrons arriving at each scintillator pixel, as well as the depth distribution of BSEs as a function of their exit energy are used as input parameters for a dynamical channeling simulation.

The dynamical scattering portion of the pattern formation is modeled using the Bloch wave approach [1] and the back-scatter probability is integrated over a depth range $z_{0}(E)$, determined from the MC simulations. If we use $\lambda(E, z)$ to represent the fraction of BSEs that exit the sample with escape energy $E$ from a depth $z$ in the sample and reach the scintillator, then the thickness-integrated BSE yield for a given exit wave vector $\mathbf{k}$ and an escape energy $E$ can be expressed as

$$
\mathcal{P}\left(\mathbf{k}, E, z_{0}(E)\right)=\sum_{i} \frac{Z_{i}^{2} D W_{i}}{z_{0}(E)} \int_{0}^{z_{0}(E)} \lambda(E, z)\left|\Psi\left(\mathbf{r}_{i}\right)\right|^{2} \mathrm{~d} z ;
$$

in this expression, the summation goes over all the atom sites in the unit cell. Each site is characterized by its atomic number $Z_{i}$ and a Debye Waller factor $D W_{i}$ that accounts for atomic vibrations. The MC simulation provides the weight factors that determine, for each scintillator pixel, what the energy distribution is of the BSEs that reach that pixel. Carrying out a series of dynamical simulations as a function of the escape energy, and combining the resulting patterns with the BSE energy weight factors then allows for the simulation of energy-weighted EBSD patterns [2].

To facilitate the computation of these energy-weighted EBSD patterns, we have introduced an areapreserving mapping from the orientation hemisphere of beam directions to a uniform square grid, using a modified Lambert projection. The dynamical simulation produces a series of Lambert projections as a function of escape energy, and each individual EBSD pattern is extracted from this "master EBSD map" by means of bi-linear interpolation on the modified Lambert projection map. Fig. 1 shows an example Lambert projection for $\mathrm{Ni}$ at $30 \mathrm{kV}$. The direction cosines of each scintillator pixel with respect to the local crystallographic reference frame determine which point of the Lambert map contributes to that pixel; the energy weight factors from the MC simulation are then combined with the energydependent Lambert map to determine the total electron count for each scintillator pixel. Examples of the resulting energy-filtered scintillator patterns are shown in Fig. 2. Our simulation results are in agreement with energy-filtered EBSD observations by Deal et al. [3]. These patterns must then be convolved with the camera point spread function to obtain the final EBSD pattern [4]. 


\section{References}

[1] Winkelmann, A. et al., Ultramicroscopy, 107 (2007), 414-421.

[2] Callahan, P.G. and De Graef, M., Microscopy and Microanalysis, under review (2013).

[3] Deal, A. et al., Ultramicroscopy, 108 (2008), 116-125.

[4] Research supported by ONR-N00014-12-1-0075.

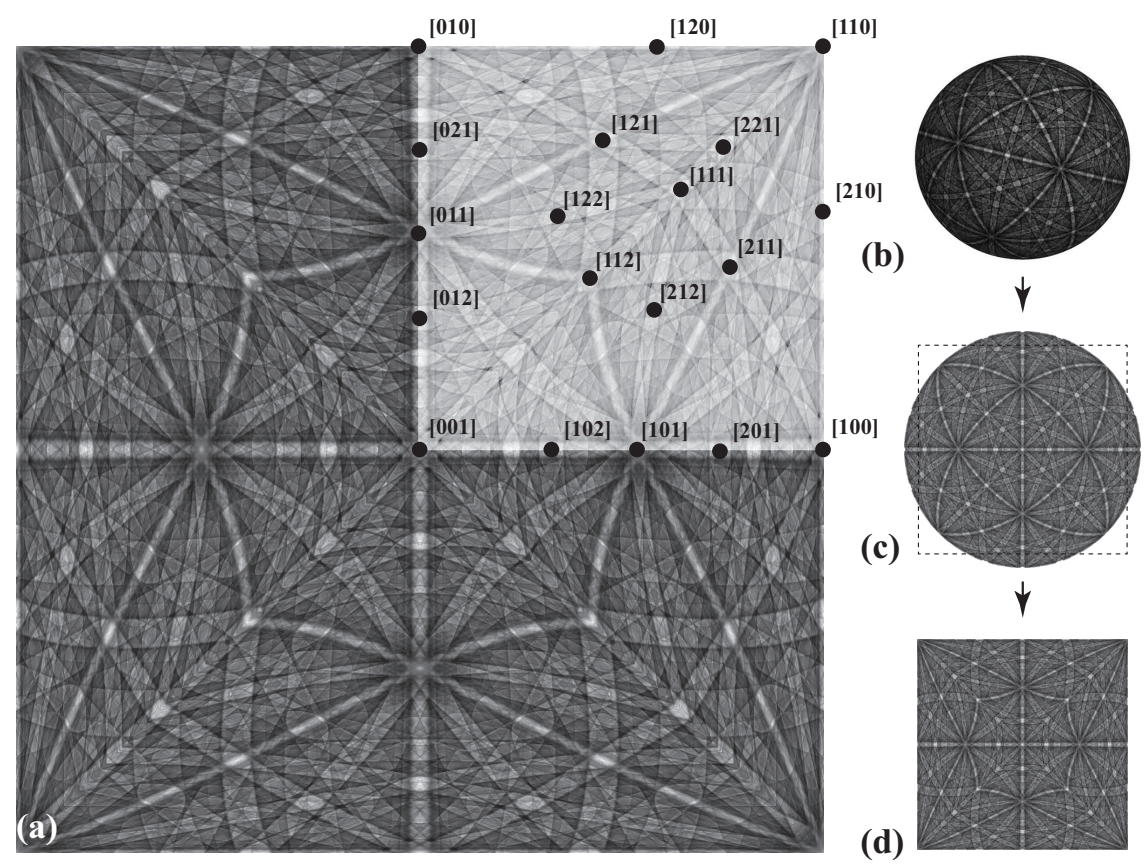

Figure 1. (a) EBSD master pattern for Ni at $30 \mathrm{kV}$; (b) spherical projection; (c) Lambert projection onto circle; (d) equal-area circle-to-square projection.

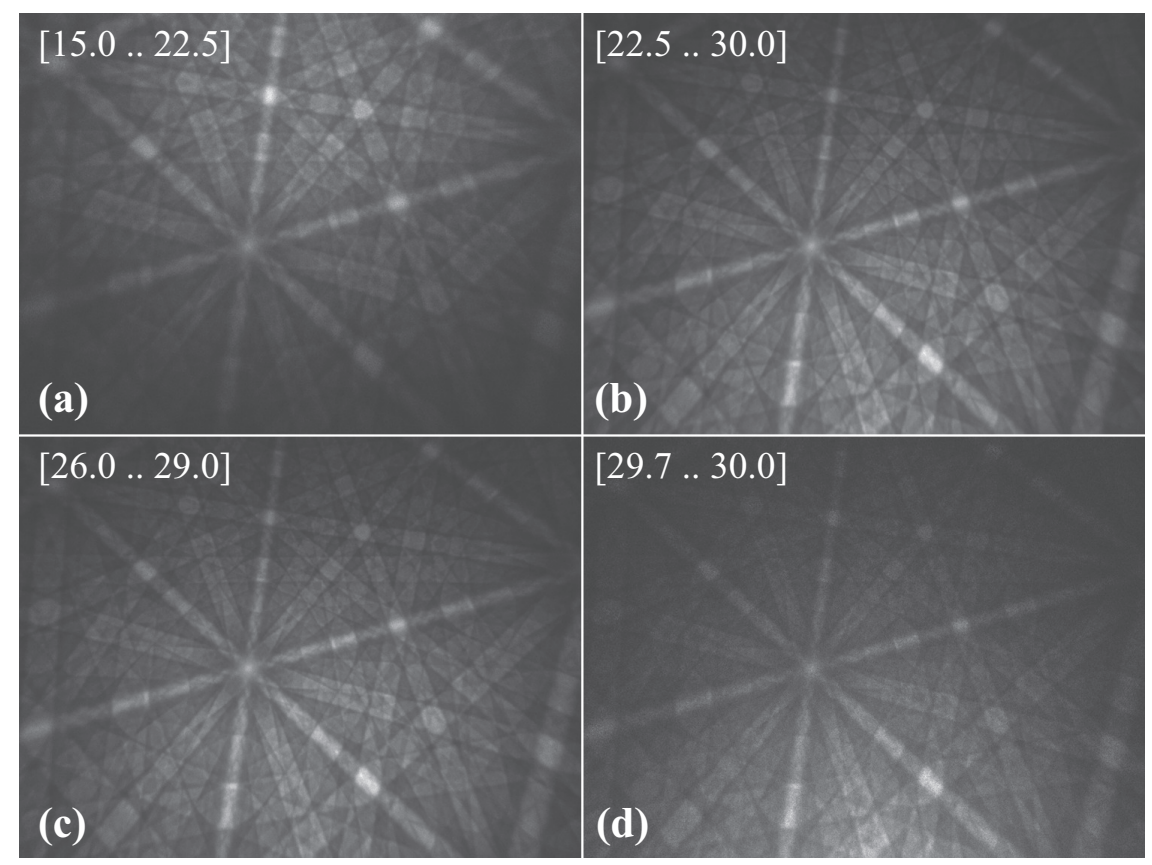

Figure 2. Energy-filtered scintillator patterns for the indicated energy windows. 